\title{
Undescended Scapula
}

\author{
Shadrick G Lungu \\ Consultant Orthopaedic Surgeon \\ South Hospital, Konkola Copper Mines Plc \\ Chingola, Zambia, \\ Tel.: +260-212-350653, Fax: +260-212-351019 \\ Shadrick.lungu@kcm.co.zm
}

\section{INTRODUCTION}

This is a congenital elevation of the scapula, also called Sprengel's deformity ${ }^{1,2,3,4}$. Many different names have been used to describe the same condition including undescended scapula. Eulenberg first described this condition in $1863^{2,3}$. In the late 1800 Otto Gerhard Karl Sprengel did a lot of work on the same condition and the deformity was named after him. Vitoirio Putti, in the early 1900 also did a lot of work on the shoulder ${ }^{3}$.

The deformity results from failure or partial descent of the scapula from the $C_{5} T_{1}$ to the $T_{2} T_{7}$ at birth and in adult life $\mathrm{e}^{2,4}$. The failure of descent is thought to be due to increased intra-uterine pressure (in utero), abnormal scapulo-spinal articulation (through an omovertebral bone), and defective musculature in the shoulder area and an arrest in development resulting in muscular tension. The affected muscles undergo degeneration, necrosis and fibrosis, resulting into contracture formation ${ }^{2}$.

This paper tries to highlight the management approach to this rare condition. The severity, function and cosmesis of the shoulder will influence the final management of the condition.

\section{Case Report}

A five year old boy was bought by his mother to our orthopaedic specialist clinic with history of a deformed left shoulder with the head/neck tilted towards the left and that he was unable to lift (abduct) his arm above the same shoulder. The deformity was noticed at the age of one year. He is the "first" and only child. There is however, history of the mother having a miscarriage prior to our patient's conception. The antenatal and postnatal history in this particular patient was uneventful. He was born in a hospital and cried well at birth.

The child underwent hydrocelectomy on the left side in 2001 when he was two years old. The family history did not reveal anything significant apart from the fact that the mother has asymmetry of the lower part of the face due to a lower jaw deformity.

On physical examination, the boy had appropriate stature for his age and he held his head tilted towards the left side. The deformity was noticeable even when clothed. General examination reviewed nothing significant except for an irregularly irregular radial pulse. His blood pressure was within normal range.

Local examination reviewed a webbed neck on the left side and an elevated shoulder on the same side. The left scapula was higher than the right and the inferior angle more pronounced. He had limited range of motion, particularly with abduction, in the left shoulder. He was unable to abduct the arm above 70 degrees.

In addition the patient had borderline cardiomegaly. Ultrasonography of the Genito-urinary system showed a right pelvic kidney and a hypoplastic, normal positioned, left kidney. The renal function test results were normal

The radiological investigations (fig.1) revealed a hypoplastic left scapula which was obviously elevated relative to the normal right one. 


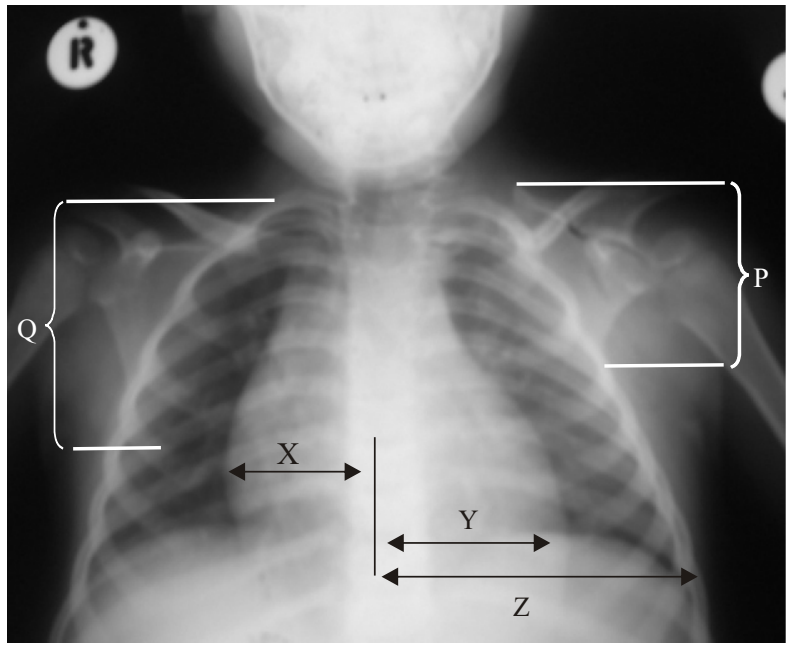

Figure 1: Pre operative Chest Radiograph: (a) Border line cardiomegaly $[(x+y)>z]$; (b) Hypoplastic scapula $P<Q$; Left shoulder elevated in relation to the contralateral side.

The parent was given the options of leaving the child alone or having him operated on for cosmetic reasons. She opted for surgery and the patient was operated on 12 February 2003. The operation was a soft tissue release and repositioning of the scapula using the Woodward procedure with morselisation of the ipslateral clavicle.

Post operatively, the shoulder was "strapped" with a crepe bandage. The straps run over the operated shoulder obliquely over the chest to the upper right abdomen and around the upper part of the abdomen (like a sergeant's belt). This was to help keep the position of the scapular to the required level. The child started his shoulder physiotherapy three days post operation. This largely involved internal and external rotation of the arm. Slowly other movements were introduced.

The post operative chest radiograph in fig. 2 shows a considerable downward "movement" of the left scapula and the morselisation of the left clavicle.

Recovery from surgery was uneventful. He was discharged ten days after the operation and was followed in the specialist clinic, during which time he continued with physical therapy.

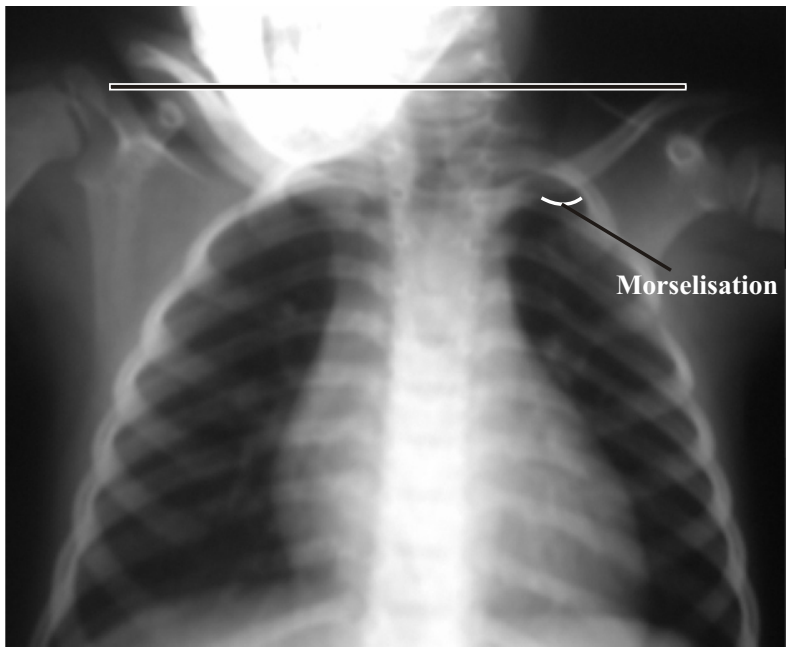

Figure 2: Post operative Chest radiograph: Level coracoid processes, left is below the horizontal line

\section{DISCUSSION}

In Sprengel's deformity, the scapula lies more superiorly than it should in relation to the thoracic cage and usually is hypoplastic and misshapen ${ }^{1,2,3}$.

This is the most common shoulder deformity ${ }^{2}$. Females are affected more than males (3:1) and the left shoulder more than the right one ${ }^{2}$. Literature reviews both genetic as well as familial basis ${ }^{2,4}$. The familial form, usually autosomal dominant inheritance, is called Corno's disease ${ }^{2,4}$.

In 70 per cent of patients with Sprengel's deformity have other associated anomalies like Klippel-Feil syndrome ${ }^{1,2,3,4}$. Other associated congenital anomalies include: absence or fusion of ribs, cervical rib, congenital scoliosis with hemiveterbrae and cervical spina bifida. Also seen associated with this condition are: clavicular abnormalities, syringomelia, paraplegia, and cardiac anomalies like ventricular septal defect, artrial septal defects and kidney malformations ${ }^{1,2}$. Our patient had congenital hydroceol and a pelvic kidney on the left side while the other kidney was hypoplastic. He also had a borderline cardiomegaly. An ultrasound scan and echo were requested to be done on the patient, but the parent was not willing, and did not therefore consent to the above two investigations. This case, however, seems to be in concordance with the literature review. It is therefore important to do exhaustive investigations in a patient with suspected 
of Sprengel's deformity.

Cavendish classification of the deformity scores the severity of the deformity ${ }^{2}$.

$\begin{array}{ll}\text { Grade I } & \text { Very mild } \\ \text { Grade II } & \text { Mild } \\ \text { Grade III } & \text { Moderate } \\ \text { Grade IV } & \text { Severe }\end{array}$

In Grade I the shoulders appear symmetrical when clothed which in itself is very mild; Grade II the superiomedial angle of the scapula is visible as a lump in the web on the neck, the deformity in this grade is mild; In Grade III the deformity is moderate and is easily visible and the shoulder is elevated 2-5 $\mathrm{cm}$; Grade IV is the severe form of the deformity, where the superior angle is near the occiput and this is associated with severe webbing of the neck ${ }^{1,2}$.

Our patient's deformity was of Grade III. When the deformity is mild, however, no treatment is indicated $^{1,2,3,4}$. The general goals of treatment therefore are to correct the deformity, improve the function and improve the cosmesis and above all physical therapy to maintain the range of motion and improve strength is a requirement. There are, however, factors that must be considered before surgery. Cosmesis will be considered in patients with a Cavendish Grade III or IV and therefore will be the indication for surgery. In the presence of omovertebral bone or scapulothoracic fibrous adhesions, surgery will be indicated to improve on the function by resection of the above stated abnormality $^{2}$. Associated abnormalities like scoliosis must be dealt with at the same time ${ }^{2}$. Literature also reviews that when the deformity is more severe, surgery may be indicated, depending on the age of the patient and severity of any associated deformities. The age, however, of the patient at operation is crucial and controversial ${ }^{1,2}$ The most agreed age is between 3 to 8 years of age $e^{1,2,4}$ However, the earlier surgery is performed after 3 years of age, the better the results, because as the child grows the operation becomes more difficulty ${ }^{1}$. The results of surgery occasionally are disappointing because the deformity is never simply elevation of the scapula ${ }^{1}$ it is always complicated by malformations and contractures of the soft tissue structures of the region. In the older children an attempt to bring the scapula inferiorly to its normal level can injure the brachial plexus ${ }^{1}$.

Numerous operations have been described to correct the Sprengel's deformity ${ }^{1,4}$. In our patient we performed the Woodward procedure with morselization of the ipsilateral clavicle ${ }^{1}$. During the operation, the ipslateral clavicle is exposed subperiosteally and divided $2 \mathrm{~cm}$ from each end and the removed part is then cut into small pieces (morcellate) and replaced in the intact periosteal tube, which is later closed with interrupted sutures ${ }^{1}$. The brachial plexus palsy is the most severe complication of the surgery for Sprengel's deformity. To avoid brachial plexus injury, several authors have recommended morselization of the clavicle on the ipislateral side as a first step in the operation of Sprengel deformity ${ }^{1}$. This is not a routine part of surgical treatment but is recommended in severe deformity or in children who show signs of brachial plexus palsy after surgical correction ${ }^{1}$. Greiteman et al., however, routinely perform an osteotomy of the clavicle to prevent this complication ${ }^{1}$.

After the above stated procedure in the supine position, the patient was then placed in a prone position on the operating table, prepared and draped both shoulders so that the involved shoulder girdle and the arm could be manipulated and the uninvolved scapula can be inspected in its normal position.

A mid line incision was made from the spinous process of the first cervical vertebra distally to that of the ninth thoracic vertebra. The skin and subcutaneous tissue were undermined laterally to the lateral border of the scapula. Then the lateral border of the trapezius muscle was identified in the distal part of the mid-line incision and by blunt dissection separated it from the underlying latissimus dorsi muscle. And by sharp dissection the fascial sheath of origin of trapezius from the spinous processes. The origins of the rhomboideus major and minor muscles were identified and sharply dissected form the spinous processes. The rhomboids and superior part of the trapezius were separated from the muscle of the chest wall anterior to them. The whole freed sheet was retracted laterally and scapula freed from the chest wall. This patient did not have any omovertebral bone connecting the scapula to the spine. The final part of 
the soft tissue release was the dividing of the narrow attachment of the trapezius at the level of the fourth vertebra. The scapula was then displaced along with the attached sheet of muscles distally until its spine was at the same level as that of the opposite side. While holding the scapula in this same position, a strong non-absorbable stitch was applied from under surface of the scapula to the chest wall. And the aponeuroses of the trapezius and rhomboids were reattached to the spinous processes at a more inferior level. The subcutaneous and skin closure was finally done.

\section{After treatment}

It is recommended that a velpeau bandage to be applied and worn for two weeks ${ }^{1}$. In our patient we applied a crepe bandage over the shoulder with a downward traction across the chest and the arm strapped against the chest with the elbow flexed across the front (trunk). It is also recommended that active and passive range of motion exercises must follow this ${ }^{1}$. Our patient commenced on passive shoulder physical therapy ten days after surgery. At two weeks post operation, the patient was discharged home and followed up in the specialist clinic. Six weeks post surgery the patient was discharged from the specialist clinic.

Six years (post-op) later the shoulder is at Cavendish Grade II (see photograph below)

\section{REFERENCES}

1. Crenshaw A.H. Campbell's Operative Orthopaedics, Saint Louis, The C. V. Mosby Company, Fifth Edition, 1971, Pg 2024

2. Legeyt M.T, Dabney K. (1996) Sprengel's Deformity, Clinical Case Presentation, http://gait.aidi.udel.edu/res/695/homepage/pd ortho/educate/clincase/sprengle.htm

3. Enersen O.D, Otto Gerhard Karl Sprengel, http://www.whonamedit.com/doctor.cfm/2144. $\underline{\mathrm{html}}$

4. Kulkarni M.L Sprengel's Deformity,

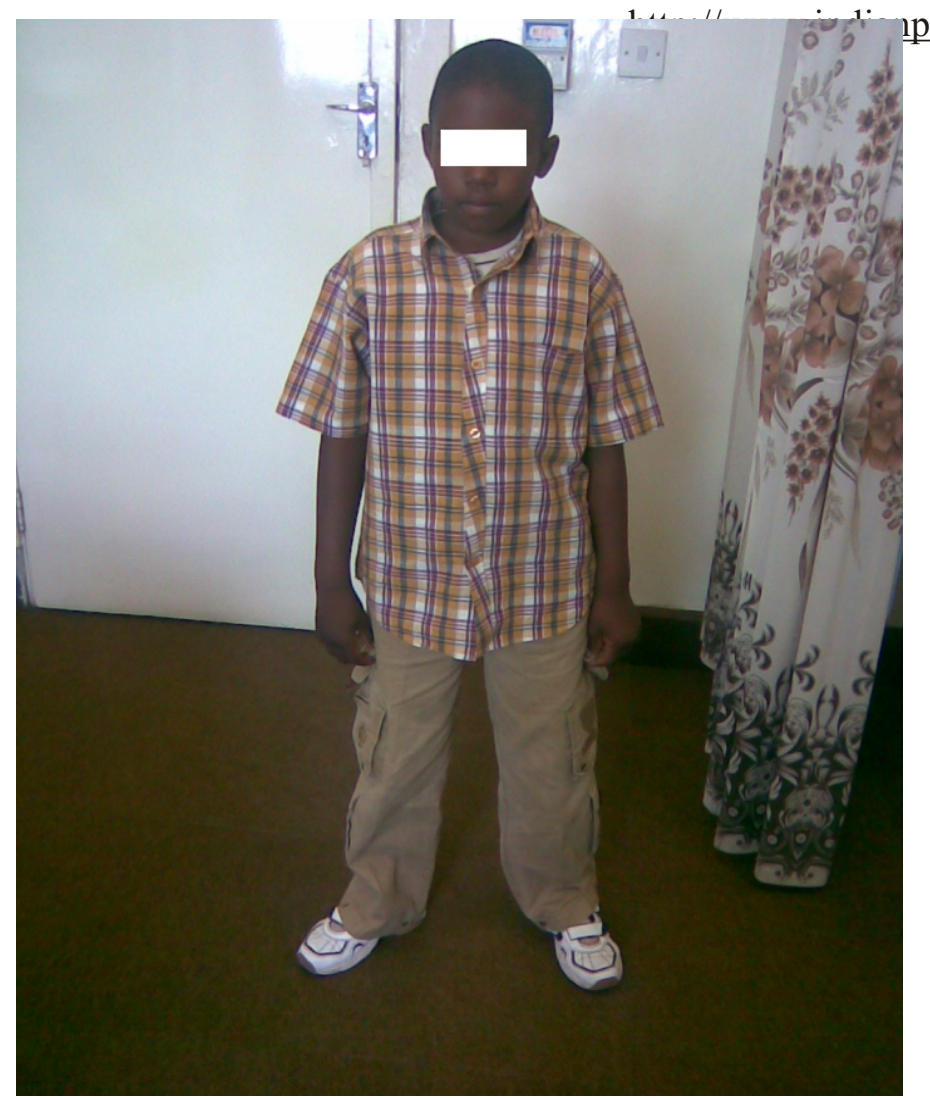

Six years after surgery (Cavendish Grade II) pediatric.net/july1994/866.p 